(2) Open Access Full Text Article

\title{
Primary adenoid cystic carcinoma of the trachea with thyroid invasion: a case report and literature review
}

This article was published in the following Dove Press journal:

OncoTargets and Therapy

13 October 2016

Number of times this article has been viewed

\author{
Dianjun $\mathrm{Qi}^{1}$ \\ Liang Feng ${ }^{2}$ \\ Jian $\mathrm{Li}^{3}$ \\ Bing $\mathrm{Liu}^{4}$ \\ Qingfu Zhang ${ }^{5}$ \\ 'Department of General Practice, \\ ${ }^{2}$ Department of Breast Surgery, \\ ${ }^{3}$ Department of Radiology, \\ ${ }^{4}$ Department of Anorectal Surgery, \\ The First Affiliated Hospital of China \\ Medical University, ${ }^{5}$ Department of \\ Pathology, The First Affiliated Hospital \\ and College of Basic Medical Sciences \\ of China Medical University, Shenyang, \\ People's Republic of China
}

Correspondence: Qingfu Zhang Department of Pathology, The First Affiliated Hospital and College of Basic Medical Sciences of China Medical University, Number 155 Nanjing North Street, Heping District, Shenyang I I000I, People's Republic of China Email qfzhang@cmu.edu.cn

\begin{abstract}
Primary adenoid cystic carcinoma (ACC) of the trachea with thyroid invasion is very rare. In this report, we present a 46-year-old man with primary ACC of the trachea with thyroid invasion. ACC invasion of the thyroid is very rare and is easily misdiagnosed. The patient sought consultation due to a 6-month history of dysphagia and associated dyspnea. A contrast-enhanced computed tomography scan obtained at the time of admission revealed bilateral thyroid masses and tracheal wall thickening. The thyroid masses were fused to the trachea and the esophagus without discernible borders, intraoperatively. Frozen pathology suggested poorly differentiated cancer, and a bilateral partial thyroidectomy was performed. Postoperative pathology revealed primary tracheal ACC with thyroid invasion. The patient died 1 month after surgery. We have also summarized the literature on the clinical presentation, diagnosis, and treatment of thyroid-invasive ACC.
\end{abstract}

Keywords: trachea, adenoid cystic carcinoma, thyroid

\section{Introduction}

Adenoid cystic carcinoma (ACC) is a relatively rare epithelial tumor that usually originates in the salivary glands, ${ }^{1,2}$ submandibular glands, and minor salivary glands, but it can also occasionally occur in the mammary glands, cervix, Bartholin glands, sinuses, skin, ${ }^{3,4}$ and even the larynx and trachea. ${ }^{5,6}$ Tracheal adenoid cystic carcinoma (TACC) is a rare, low-grade, malignant tumor that originates in the glands of the tracheal and bronchial walls. The histological structure and biological behavior of TACC are similar to those of tumors found in the salivary glands, and TACC most commonly occurs in the upper trachea, being found in the joint cartilage in the posterior aspect of the trachea. Primary TACC most frequently metastasizes to the lungs and the bone. ${ }^{7}$ It rarely involves the thyroid, and therefore, it can be misdiagnosed as thyroid cancer. In this report, we present a case of TACC involving the thyroid.

\section{Case report}

A 46-year-old man with no history of smoking or alcohol use was admitted with a 6-month history of dysphagia and associated dyspnea with worsening symptoms for 1 month. There was no obvious cause for his dysphagia and associated dyspnea. Bronchoscopy examination revealed bronchial stenosis, but no pathology studies or further treatment was performed. Soon thereafter, his symptoms worsened, and he developed cervical pain. Plain computed tomography (CT) of the trachea showed tracheal compression and displacement with slight narrowing. The subglottic tracheal 
wall was thickened (Figure 1A). The tumor did not invade the glottis (Figure 1B). Thyroid CT examination showed multiple thyroid nodules. The most prominent nodule was round and hypodense, and it was located in the right thyroid lobe (Figure 1C). The nodule measured 84 Hounsfield units (HU), unenhanced, and 95 HU with slight enhancement on a contrast-enhanced scan (Figure 1D). Examination of the rest of the head and neck showed no signs of tumor or lymphadenopathy.

During intraoperative exploration, we discovered undemarcated invasion and fusion of the right thyroid lobe to the esophagus and posterolateral aspect of the trachea. Bilateral thyroid masses were resected, and frozen pathology was obtained, showing that both the masses were poorly differentiated carcinomas (Figure 2A). Owing to tumor invasion and extensive spread, radical resection would have been difficult. Hence, we decided to perform bilateral partial mass resections after a discussion with the family. Pathology showed that the tumor tissue had no capsule formation and grew in an infiltrative manner, invading the surrounding thyroid follicles. Tumor cells were cribriform or tubular, and they were distributed in a solid funicular pattern. The masses consisted of double-layer structures, with glandular epithelium as the inner layer and basal cells forming the outer layer. The cribriform structure was composed of differentially sized scattered or integrated smooth cellular islands, with a central, round space filled with slightly basophilic mucoid material (Figure 2B). There was little cell atypia, and mitosis was rare. Interstitial hyalinization was present, but no obvious nerve infiltration was seen. Postoperative immunohistochemistry showed that the ductal epithelial cells were positive for pan-cytokeratin (CK), CK7 (Figure 2C), and CD117 (Figure 2D). The myoepithelial cells were positive for vimentin, S-100, P40 (Figure 2E),
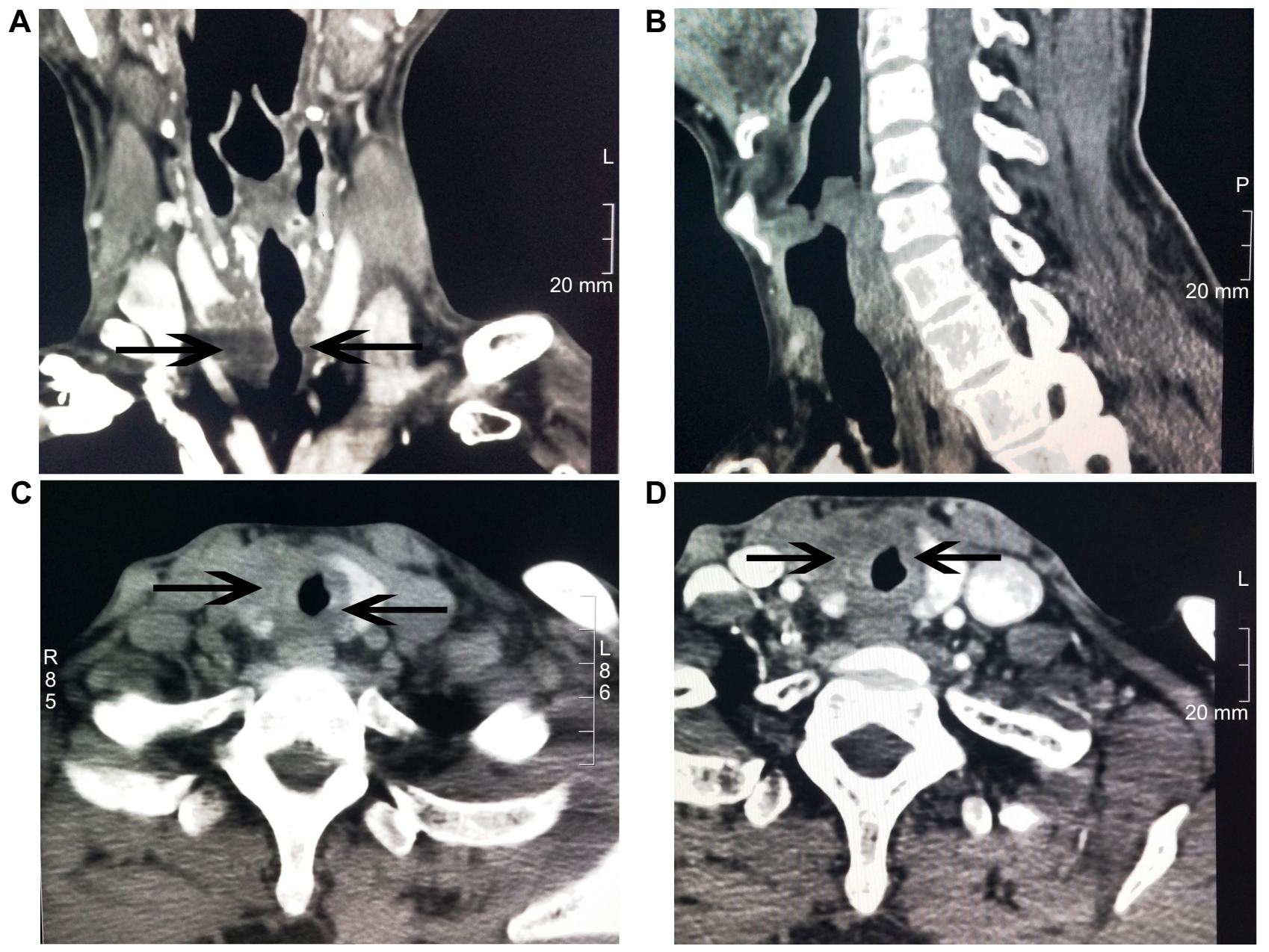

Figure I CT images of the trachea and thyroid.

Notes: (A) A plain CT scan of the trachea shows tracheal compression and displacement. (B) The tumor did not invade the glottis. (C) A plain thyroid CT scan reveals multiple bilateral thyroid nodules. (D) An enhanced thyroid CT scan displays thyroid nodules that are enhanced slightly.

Abbreviations: CT, computed tomography; HU, Hounsfield units. 

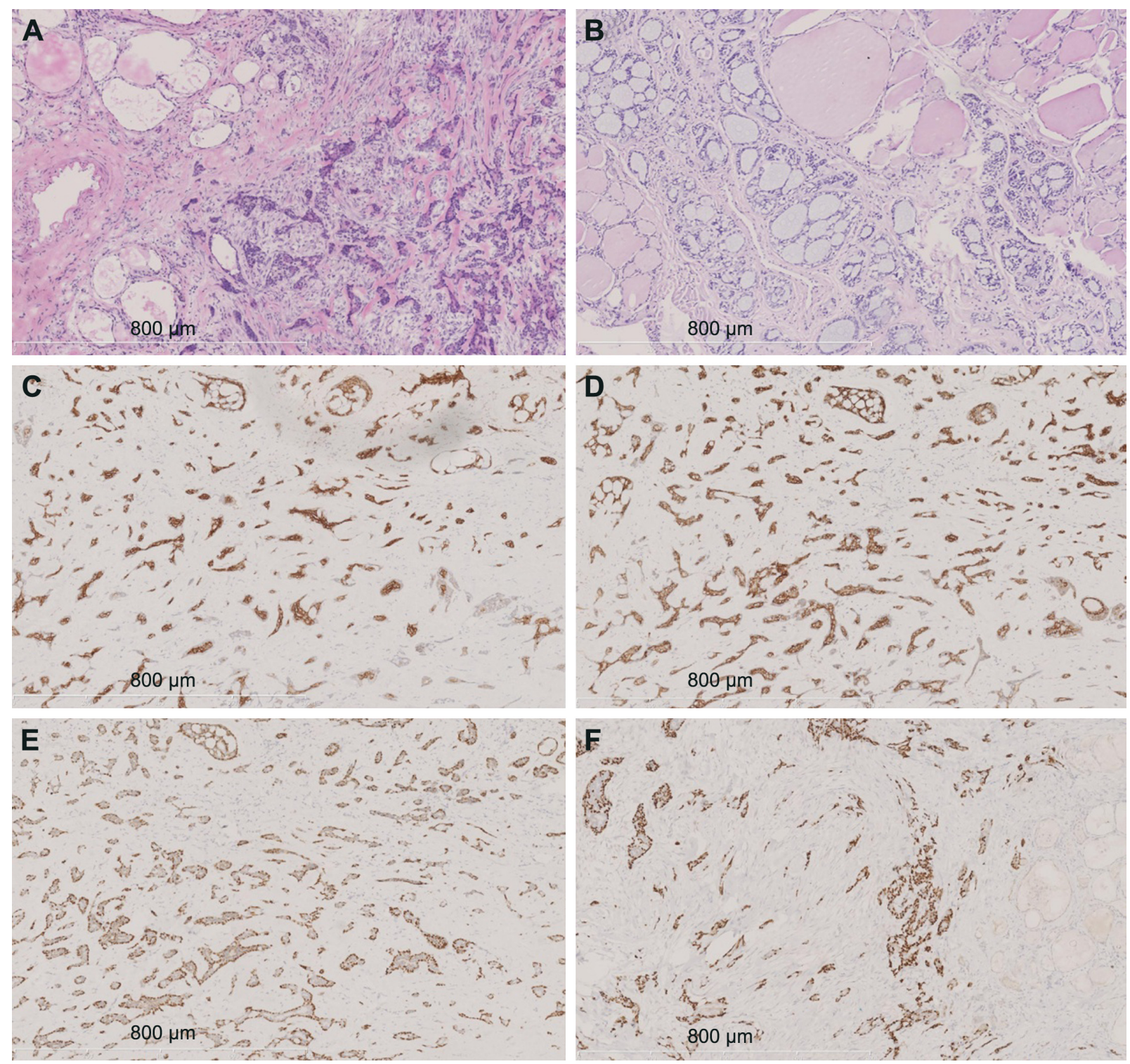

Figure 2 Immunohistochemical staining findings.

Notes: (A) Frozen section analysis shows tumor cells in an irregular funicular distribution with invasive growth (100x). (B) The tumor infiltrates surrounding thyroid follicles in a cribriform and tubular distribution, and it is filled with slightly basophilic mucoid material consisting of a double-layer structure (hematoxylin and eosin staining, I00 $\times$ ). (C) Positive CK7 staining in glandular epithelial cells (I00X). (D) Positive CDII7 staining in glandular epithelial cells (I00X). (E) Positive p40 staining in basal cells (I00X). (F) Positive p63 staining in basal cells (100x).

and P63 (Figure 2F). The Ki67 cellular proliferation index was approximately $10 \%$. The cells were negative for thyroid transcription factor-1, CD56, calcitonin, synaptophysin, and thyroglobulin. Based on imaging and histopathological morphology findings, the patient was diagnosed with primary TACC with thyroid invasion. The patient was discharged from the hospital 4 days after surgery. However, the patient died of cardiopulmonary failure 1 month later, at home. No postmortem autopsy was performed.

\section{Discussion}

ACC does not have a high rate of incidence. ${ }^{8}$ The average age at onset is 57.4 years, and approximately $60 \%$ of patients are women. ${ }^{9}$ Most ACCs originate in the salivary glands, and TACC, which originates in the trachea, is rare. The incidence rates of TACC are similar in both men and women. Currently, surgical resection is still the main treatment method for TACC,${ }^{10}$ and it has been shown to improve survival. ${ }^{11}$ In recent years, adjuvant treatments, such as radiotherapy and chemotherapy, have been reported to provide some treatment effect. ${ }^{12}$ Radiotherapy can be used to treat patients with inoperable ACC, but its efficacy remains uncertain. ${ }^{13,14}$

ACC is a slow-growing tumor, but metastasis often occurs, ${ }^{11,15}$ especially late metastasis up to 15 years after initial diagnosis ${ }^{16}$ and multiple metastases. ${ }^{7}$ Approximately one-third of patients with ACC will develop distant 
metastasis, with common sites being the lungs, brain, bone, and liver. ${ }^{17,18}$ There have been few reports in the English literature describing ACC involving the thyroid. Including our case, this report reviews a total of ten cases of primary ACC with thyroid invasion (Table 1). ${ }^{5,6,19-24}$ These ten cases included four male (40\%) and six female patients $(60 \%)$. Their ages ranged from 17 to 68 years, with the average age being 49.8 years. There were no current smokers among them. Unfortunately, there was no information on passive smoking for any patients. Therefore, it was impossible to assess smoking-associated risk. Of the ten ACC cases, six primary tumors originated from the bronchi, three tumors originated from the laryngeal minor salivary glands, and one tumor originated from the left parotid gland. Respiratory symptoms were the main clinical presentation for all ten patients. Four patients experienced dyspnea, three had cough, two experienced hoarseness, two developed asthma, two experienced wheezing, one had hemoptysis, one developed neck pain, and one had no symptoms at all. Of the ten cases, three involved the left thyroid lobe, six involved both the lobes, and one involved the thyroid isthmus. Metastases varied in size, with the largest being $8 \times 6 \times 5 \mathrm{~cm}$ and the smallest being $1.0 \times 0.7 \mathrm{~cm}$. Three patients had concomitant multiple metastases, which were located in the arytenoid and cricoid cartilage, lungs, muscle, and esophagus. Currently, surgery is still the main treatment for ACC involving the thyroid. ${ }^{22}$ Seven of ten patients underwent radical resection, one patient underwent local excision, and one patient did not receive any surgery. In addition to surgical treatment, three patients received radiotherapy and one patient received radiotherapy and chemotherapy. Patient survival time varied greatly after metastasis. The shortest survival time was only 1 month, and the longest was $>48$ months. The prognosis of female patients seems better than that of male patients. Only one male case report provided the time interval between diagnosis and death. In this case, the patient died within a month after diagnosis, while all six female patients lived for $>3$ months.

TACC involving the thyroid is very rare, and to the best of our knowledge, our case is the sixth report in English literature. TACC involves the thyroid mainly through invasion via direct extension, and few cases present with metastasis. ${ }^{20}$ Among the six current reported cases of TACC involving the thyroid, the average patient age was 49 years, and there were two male and four female patients. Respiratory symptoms, including asthma, cough, dyspnea, dysphagia, hoarseness, hemoptysis, wheezing, and neck pain, were the main clinical presentations, all of which lack

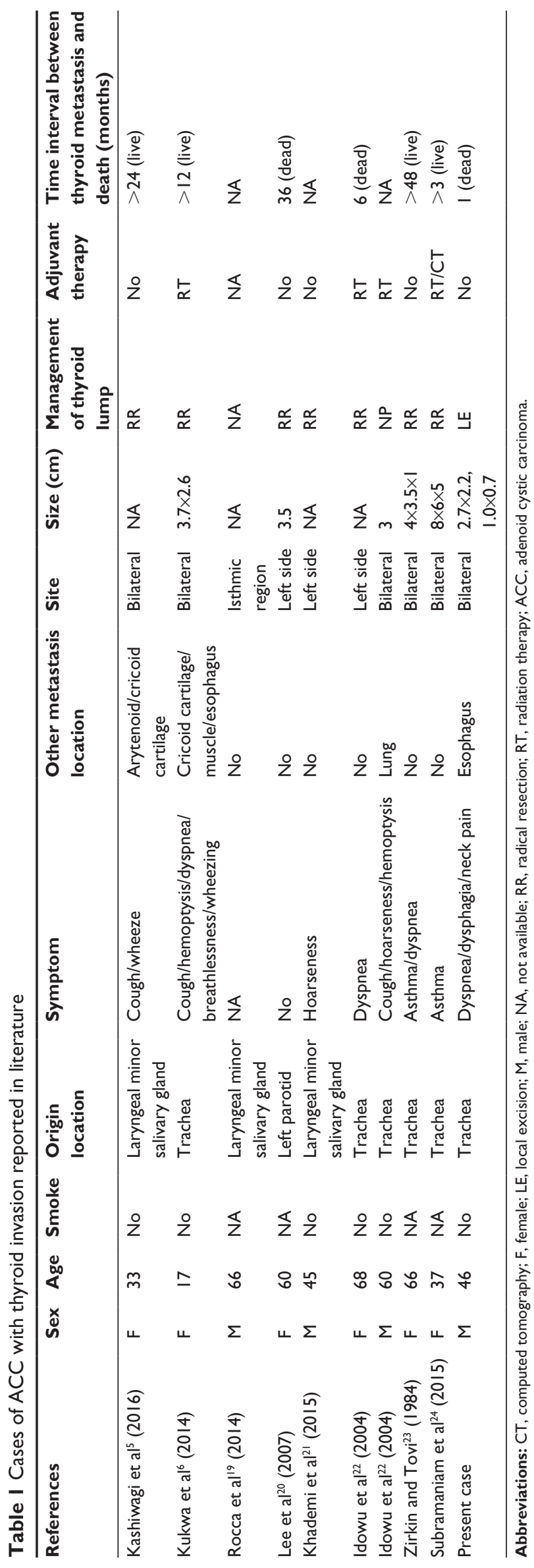


specificity. Five cases involved bilateral metastasis, with the largest being $8 \times 6 \times 5 \mathrm{~cm}$. This may be due to the nonspecific symptoms, leading to late diagnosis, bilateral involvement, and larger tumor masses at the time of diagnosis. Three out of the six cases had multiple organ involvement other than the trachea, and five out of six patients elected for surgical treatment, with four choosing radiotherapy and one opting for chemotherapy. Surgical options depended on the primary tumor location as well as tissue and organ involvement. The most common surgical procedures include primary tracheal tumor resection, trachea reconstruction, and distal metastases resection. For patients with esophageal involvement, esophageal reconstruction was performed at the same time.

As the incidence of TACC is relatively low, thyroid involvement is even rarer. ${ }^{22,23}$ In addition, owing to the close anatomical proximity of the trachea and the thyroid, it is difficult to accurately determine the nature of the tumor preoperatively, thereby resulting in misdiagnosis. In our case, a preoperative $\mathrm{CT}$ scan showed merging of the thyroid mass with the tracheal mass, but it did not reveal the relationship between the two. It was only discovered intraoperatively that the thyroid mass had fused with the trachea and the esophagus. Therefore, we suggest that a CT scan and fine-needle aspiration be completed prior to this type of surgery, which would facilitate an earlier diagnosis. ${ }^{20}$

\section{Conclusion}

Any extrathyroid mass involving the thyroid is extremely rare. For patients with a thyroid mass, the presence of tracheal lesions or a history of ACC suggests the possibility of ACC with metastasis to the thyroid. A comprehensive investigation should be performed simultaneously to determine any multiorgan involvement. Comprehensive imaging and pathological examination can facilitate a clear preoperative diagnosis and the early development of a sound treatment plan.

\section{Acknowledgments}

This work was supported by the Natural Science Foundation of Liaoning Province in China (Numbers L2015598 and 201602877). The patient provided written informed consent for the publication of the data and associated images.

\section{Disclosure}

The authors report no conflicts of interest in this work.

\section{References}

1. Laurie SA, Ho AL, Fury MG, Sherman E, Pfister DG. Systemic therapy in the management of metastatic or locally recurrent adenoid cystic carcinoma of the salivary glands: a systematic review. Lancet Oncol. 2011;12(8):815-824.
2. Chaudhry AP,LeiferC, CutlerLS, Satchidanand S, Labay GR, Yamane GM. Histogenesis of adenoid cystic carcinoma of the salivary glands. Light and electronmicroscopic study. Cancer. 1986;58(1):72-82.

3. Lawrence JB, Mazur MT. Adenoid cystic carcinoma: a comparative pathologic study of tumors in salivary gland, breast, lung, and cervix. Hum Pathol. 1982;13(10):916-924.

4. Qing S, Zhou K, Liu X, Li X, Deng F, Ma Y. Primary pulmonary adenoid cystic carcinoma: clinicopathological analyses of 12 cases. Int $J$ Clin Exp Pathol. 2015;8(6):7619-7626.

5. Kashiwagi T, Kanaya H, Konno W, Goto K, Hirabayashi H, Haruna S. Adenoid cystic carcinoma of the larynx presenting with unusual subglottic mass: case report. Auris Nasus Larynx. 2016;43(5):562-565.

6. Kukwa W, Korzeń P, Wojtowicz P, et al. Tracheal adenoid cystic carcinoma mimicking a thyroid tumor: a case report. Oncol Lett. 2014;8(3): 1312-1316.

7. Alshammari A, Eldeib OJ, Eldeib AJ, Saleh W. Adenoid cystic carcinoma of the submandibular gland with rare metastasis to the sternum in a 52-year-old male. Ann Thorac Med. 2016;11(1):82-84.

8. Spiro RH, Huvos AG, Strong EW. Adenoid cystic, carcinoma of salivary origin. A clinicopathologic study of 242 cases. Am J Surg. 1974; 128(4):512-520.

9. Ellington CL, Goodman M, Kono SA, et al. Adenoid cystic carcinoma of the head and neck: incidence and survival trends based on 1973-2007 surveillance, epidemiology, and end results data. Cancer. 2012;118(18): 4444-4451.

10. Kanematsu $\mathrm{T}$, Yohena $\mathrm{T}$, Uehara $\mathrm{T}$, et al. Treatment outcome of resected and nonresected primary adenoid cystic carcinoma of the lung. Ann Thorac Cardiovasc Surg. 2002;8(2):74-77.

11. Bradley PJ. Adenoid cystic carcinoma of the head and neck: a review. Curr Opin Otolaryngol Head Neck Surg. 2004;12(2):127-132.

12. Allen AM, Rabin MS, Reilly JJ, Mentzer SJ. Unresectable adenoid cystic carcinoma of the trachea treated with chemoradiation. $J$ Clin Oncol. 2007;25(34):5521-5523.

13. Bonner Millar LP, Stripp D, Cooper JD, Both S, James P, Rengan R. Definitive radiotherapy for unresected adenoid cystic carcinoma of the trachea. Chest. 2012;141(5):1323-1326.

14. Vikram B, Strong EW, Shah JP, Spiro RH. Radiation therapy in adenoid-cystic carcinoma. Int J Radiat Oncol Biol Phys. 1984;10: 221-223.

15. Sung MW, Kim KH, Kim JW, et al. Clinicopathologic predictors and impact of distant metastasis from adenoid cystic carcinoma of the head and neck. Arch Otolaryngol Head Neck Surg. 2003;129(11): 1193-1197.

16. Matsuba HM, Simpson JR, Mauney M, Thawley SE. Adenoid cystic salivary gland carcinoma: a clinicopathologic correlation. Head Neck Surg. 1986;8(3):200-204.

17. Ko JJ, Siever JE, Hao D, Simpson R, Lau HY. Adenoid cystic carcinoma of head and neck: clinical predictors of outcome from a Canadian centre. Curr Oncol. 2016;23(1):26-33.

18. Sa YJ, Sim SB, Kim TJ, Moon SW, Park CB. Late-developing tongue adenoid cystic carcinoma after pulmonary metastasectomy: a case report. World J Surg Oncol. 2014;21:102.

19. Rocca BJ, Barone A, Ginori A, Ambrosio MR, Disanto A. Unusual presentation of metastatic adenoid cystic carcinoma: a challenge in aspiration cytology of the thyroid. Pathologica. 2014;106(4):342-344.

20. Lee MW, Batoroev YK, Odashiro AN, Nguyen GK. Solitary metastatic cancer to the thyroid: a report of five cases with fine-needle aspiration cytology. Cytojournal. 2007;4:5.

21. Khademi B, Negahban S, Aledavood A, et al. An unusual thyroid mass Am J Med. 2015;128(10):e29-e30.

22. Idowu MO, Reiter ER, Powers CN. Adenoid cystic carcinoma: a pitfall in aspiration cytology of the thyroid. Am J Clin Pathol. 2004;121(4): 551-556.

23. Zirkin HJ, Tovi F. Tracheal carcinoma presenting as a thyroid tumor. J Surg Oncol. 1984;26(4):268-271.

24. Subramaniam T, Lennon P, Kinsella J, O’Neill JP. Laryngeal preservation in managing advanced tracheal adenoid cystic carcinoma. Case Rep Otolaryngol. 2015;2015:404586. 


\section{Publish your work in this journal}

OncoTargets and Therapy is an international, peer-reviewed, open access journal focusing on the pathological basis of all cancers, potential targets for therapy and treatment protocols employed to improve the management of cancer patients. The journal also focuses on the impact of management programs and new therapeutic agents and protocols on

patient perspectives such as quality of life, adherence and satisfaction. The manuscript management system is completely online and includes a very quick and fair peer-review system, which is all easy to use. Visit http://www.dovepress.com/testimonials.php to read real quotes from published authors.

Submit your manuscript here: http://www.dovepress.com/oncotargets-and-therapy-journal 